\title{
Prevalence of Temporomandibular Disorders and its Correlation with Stress and Salivary Cortisol Levels among Students
}

\author{
Swapna Bettanapalya Venkatesh ${ }^{1}$, Smitha Sammith Shetty ${ }^{2}$,, Vignesh Kamath ${ }^{10}$
}

\begin{abstract}
${ }^{1}$ Department of Prosthodontics, Faculty of Dentistry, Melaka Manipal Medical College, Manipal Academy of Higher Education, Manipal, Karnataka, India.

${ }^{2}$ Department of Oral Pathology, Faculty of Dentistry, Melaka Manipal Medical College, Manipal Academy of Higher Education, Manipal, Karnataka, India.
\end{abstract}

Correspondence: Dr Vignesh Kamath, Assistant Professor, Department of Prosthodontics, Faculty of Dentistry, Melaka Manipal Medical College, Manipal Academy of Higher Education, Manipal, Karnataka, 576104, India. E-mail: vignesh.kamath@manipal.edu

\section{Academic Editor: Burak Buldur}

Received: 04 June 2020 / Review: 02 October 2020 / Accepted: 08 October 2020

\begin{abstract}
How to cite: Venkatesh SB, Shetty SS, Kamath V. Prevalence of temporomandibular disorders and its correlation with stress and salivary cortisol levels among students. Pesqui Bras Odontopediatria Clín Integr. 2021; 21:e0120. https://doi.org/10.1590/pboci.2021.029
\end{abstract}

\begin{abstract}
Objective: To evaluate the prevalence of temporomandibular disorders (TMD) in students and to evaluate if any relationship existed between the stress levels, salivary cortisol levels, and TMD. Material and Methods: A total of 348 students, 187 female, and 161 male students, participated in this cross-sectional study. Students were evaluated based on the Research Diagnostic Criteria for TMD. The stress levels were evaluated using the Perceived Stress Scale. The students were divided into the control and TMD groups. Salivary cortisol levels in the salivary samples were analyzed. Results: The prevalence rate of TMDs was $30.7 \%$ in the study population. Of the female students, $61 \%$ had TMD compared with $46 \%$ of male students. Muscle disorders were the most predominant disorder in $14.2 \%$ of the students with TMD. The TMD group showed significantly higher salivary cortisol and stress levels than the control group. The TMD group also showed a moderate positive correlation between cortisol and stress levels $(\mathrm{p}=0.01)$. Conclusion: The study showed a strong association between salivary cortisol levels, stress, and temporomandibular disorders. Salivary cortisol could be used as a prognostic biomarker for stress while assessing the severity of TMJ problems in stressed individuals.
\end{abstract}

Keywords: Hydrocortisone; Saliva; Temporomandibular Joint Dysfunction Syndrome. 


\section{Introduction}

Temporomandibular disorders (TMD) represent numerous problems related to temporomandibular joints, masticatory musculature, and related structures [1]. TMD is described by a typical triad of clinical signs, such as TMJ sounds, TMJ pain or masticatory muscle pain, and deviations or limitations in jaw movements [2]. TMD's symptoms increase in adolescence, become prominent during middle age, and gradually reduce later in life $[3,4]$.

The diagnosis of TMD is always established by the examination of signs and symptoms; however, the presence of symptoms is variable. Few epidemiological studies have been conducted to evaluate the prevalence of TMD in patients and healthy individuals. Furthermore, the prevalence rate of $16-59 \%$ in the common population has been reported [5-7]. Solberg et al. [8] reported that TMD symptoms existed in approximately $26 \%$ of college students. A higher prevalence of TMD symptoms in females has been reported [9]. Zulqarnain et al. [10] reported a prevalence of $12 \%$ in female college students.

Many studies have used self-administered questionnaires to evaluate the prevalence of TMDs in the general population [9-13]. The Research Diagnostic Criteria for Temporomandibular Disorders (RDC/TMD) was first recommended by Dworkin and Leresche [11]. The RDC/TMD detects the complete relationship between the psychological and physical dimensions of chronic pain. It provides a correct measurement of signs and symptoms in TMD (Axis I) and the associated psychosocial and psychological factors (Axis II) [12]. The RDC/TMD has been verified for validity and reliability for Axis I and II. It is universally accepted because of its good to excellent results [11-15].

TMD has a multifactorial etiology, but the role of individual etiological factors remains controversial [16]. Patients with TMD present with a range of behavioral and psychosocial features and psychological stress is an important triggering factor [17]. Stress is a condition of psychological or physiological pressure caused by different stimuli. Socioeconomic factors, changes in the lifestyle, competitive workloads, and emotional disturbances lead to increased stress levels in the existing scenario [18]. The mechanism of stress response leads to two chemical sequences. A quick response is facilitated by the adrenaline and noradrenaline hormones, and a slow response is facilitated by the cortisol hormone. The cortisol hormone is generally considered as a stress level indicator [19].

Patients with higher stress levels are more prone to bruxism and TMJ disorders and generally, excessive stress has several implications on physical and mental health [20]. Frequent exposure to stressful conditions can increase cortisol levels, which can have harmful effects on health. Salivary cortisol level is a valid indicator for the plasma cortisol concentration [21]. Like plasma cortisol, the cortisol concentration in the saliva is a dependable stress marker [22]. This study aimed to measure the prevalence of TMDs in dental and medical students using the RDC/TMD Axis I questionnaire. The second objective was to assess the stress and saliva cortisol levels in students with TMD and to compare the results with students without TMD. The hypothesis established was that students with TMD did not present with any changes in stress and salivary cortisol levels.

\section{Material and Methods}

Study Design and Ethical Clearance

A cross-sectional study was conducted after obtaining ethical approval from the Institutional Ethical Committee, Kasturba Medical College, Manipal (IEC196/2019 dated 13/3/2019). This study was registered in the Clinical Trial Registry India (CTRI/2019/05/019368). The participants were explained in detail 
regarding the importance of the study, and written informed consent was obtained from all the students who were willing to participate in the study.

\section{Sample Size}

A total of 348 dental and medical students between the age group of 18 to 23 years, from Melaka Manipal Medical College, Manipal, participated in this study. The sample size was calculated based on the prevalence rate of a previous study [23].

Inclusion and Exclusion Criteria

The following inclusion criteria were established: students voluntarily willing to participate in the study; normal sleep habits and complete dental arches (erupted third molars were not required). The following exclusion criteria were adopted: smoking habit; any history of systemic illness; a history of orthodontic treatment; use of any dental prosthesis; dental pain and any facial pain interfering with the diagnosis of TMD

\section{Sleep Assessment}

Assessment of normal sleep habits was performed by considering the sleep timing, which should be regular and conventional with no sign of sleep disruption [24].

\section{TMD Assessment}

A modified version of Axis I questionnaire of the RDC/TMD was used, which facilitated the establishment of the TMD patterns and categorized the students into two groups: those without TMD and those with TMD. The RDC/TMD version 1 categorizes patients based on their diagnosis into the following three groups: muscle disorders (Group I), disk displacement (Group II), and arthralgia, osteoarthritis, or osteoarthrosis (Group III) [11].

A single examiner performed the clinical examination following the method mentioned in RDC/TMD, and the findings were recorded. The range of mandibular movements (mouth opening, protrusion, and lateral movements) and mandibular movements were recorded by the Okeson method [19]. Palpation of the muscles and TMJ was performed to record pain or tenderness. The muscles were palpated bilaterally by application of digital pressure. Extraoral palpation of the temporalis, masseter, and medial pterygoid muscles was performed, and the lateral pterygoid and medial pterygoid were palpated intraorally. The muscles were palpated bilaterally on each side by unilateral palpation with firm pressure exerted by one finger (intra-oral muscles) or two fingers (extra-oral muscles). TMJ sounds for vertical motion, lateral excursions, and protrusion were recorded using a stethoscope. Scoring was performed following the clinical examination, and the diagnosis was established using RDC/TMD Axis 1.

The second part of this study was to measure the salivary cortisol and stress values between the two groups: a control group consisting of students without TMD disorders and a case group consisting of students with TMD disorders diagnosed using RDC/TMD criteria. The sample size consisted of 40 participants. Simple random sampling by the lottery method was performed for the selection of 20 students with TMD in the TMD group and 20 students without TMD as controls.

Perceived Stress Scale 
The Perceived Stress Scale (PSS) questionnaire consisting of 10 questions was modified and administered to the participants and they were instructed to answer on the same day as the collection of the saliva sample [19]. The questionnaire included a few direct questions regarding the existing stress levels. Each question was rated on a 5-point scale (0, never; 1, almost never; 2, sometimes; 3, often; and 4, very often. For questions 4, 5, 7 and 8 the scores were changed as $0=4,1=3,2=2,3=1,4=0$, and final score was calculated by addition of the individual scores. The individual scores on the PSS ranged between 0 and 40 with a high score indicating a high level of perceived stress. The scores were interpreted as follows: A) Scores ranging from 0-13 indicated low stress; B) Scores ranging from 14-26 indicated moderate stress, and C) Scores ranging from 27-40 indicated high-perceived stress.

Collection and Analysis of the Saliva

Collection of saliva was done on the same day in the morning between 9.30 am to 10.00 am. The students were instructed to chew sugar-free chewing gum to stimulate the salivary flow, and $10 \mathrm{~mL}$ of saliva samples were collected in a test tube and later transferred into a sterile centrifuge tube. The salivary samples were immediately frozen at $-20^{\circ} \mathrm{C}$ and stored at the same temperature. Later, samples were thawed at $37^{\circ} \mathrm{C}$ and then centrifuged at $8,000 \mathrm{rpm}$ for $10 \mathrm{~min}$. The supernatant fluid obtained was used to estimate cortisol levels using the electrochemiluminescence immunoassay on an Elecsys immunoassay analyzer (Roche Diagnostics International Ltd, Rotkreuz, Switzerland). The cortisol saliva Elisa kit (IBL International Corp., Bottighofen, Switzerland) was used for analysis.

Statistical Analysis

Data were tabulated and statistically analyzed using the Statistical Package for Social Science version 16.0. The chi-square test was used to evaluate the prevalence of TMD among the participants. The MannWhitney $\mathrm{U}$ test analyzed the levels of stress in the two groups. The unpaired t-test evaluated the cortisol levels. A p-value of $<0.05$ was considered statistically significant. The Spearman's correlation test assessed the strength of the association between stress and salivary cortisol levels in patients with TMD.

\section{Results}

This study evaluated the prevalence of TMD in men and women with a mean age of 20.5 years. A total of 348 students responded to the questionnaire. The sample consisted of $53.7 \%$ women and $46.2 \%$ men. As seen in Table 1, the prevalence of TMD among the study population was $30.7 \%$, and female students $(57 \%)$ presented a higher prevalence of TMD than the male students (43\%). The prevalence of TMD in the comparison between the sexes was not statistically significant $(p=0.414)$.

Table 1. Prevalence of TMD according to gender.

\begin{tabular}{|c|c|c|c|c|}
\hline \multirow{3}{*}{ TMD } & \multicolumn{2}{|c|}{ Gender } & \multirow{3}{*}{$\begin{array}{l}\text { Total } \\
\text { N (\%) }\end{array}$} & \multirow{3}{*}{ p-value* } \\
\hline & Males & Females & & \\
\hline & $\mathrm{N}(\%)$ & N (\%) & & \\
\hline Yes & 46 (43.0) & $61(57.0)$ & $107(30.7)$ & 0.414 \\
\hline No & $115(47.7)$ & $126(52.3)$ & 241 (69.3) & \\
\hline
\end{tabular}

*Chi-square test.

Table 2 explains TMD's diagnosis assessed using Axis I into group I, group II, and group III. Group I disorder (myofacial pain) were the most prevalent among the participants (14.2\%). Disk displacement was 
observed in $5.6 \%$ of the participants with a reduction on the right/left/both TMJs; however, only $4.2 \%$ of students had associated pain and required treatment. Only $1.3 \%$ of the participants showed disk displacement without reduction, and $4.8 \%$ of the participants had joint disorders, such as osteoarthritis and arthralgia.

Table 2. Prevalence of the TMD Axis I.

\begin{tabular}{|c|c|c|c|}
\hline Group & Variables & $\mathbf{N}$ & $\%$ \\
\hline \multirow[t]{2}{*}{ I } & Muscle Disorders & & \\
\hline & Myofascial Pain & 40 & 11.4 \\
\hline \multirow[t]{10}{*}{ II } & Myofascial Pain with Limited Mouth Opening & 10 & 2.8 \\
\hline & Disk Displacement with Reduction & & \\
\hline & Present, Left / Right TMJ & 11 & 3.1 \\
\hline & Present, Left and Right TMJs & 9 & 2.5 \\
\hline & Disk Displacement with Reduction with Pain & & \\
\hline & Present, Left / Right TMJ & 10 & 2.8 \\
\hline & Present, Left and Right TMJs & 5 & 1.4 \\
\hline & Disk Displacement without Reduction & & \\
\hline & Present, Left / Right TMJ & 4 & 1.1 \\
\hline & Present, Left and Right TMJs & 1 & 0.2 \\
\hline \multirow[t]{3}{*}{ III } & Other Joint Disorders - Arthralgia, Osteoarthritis, Osteoarthrosis & & \\
\hline & Present, Left / Right TMJ & 10 & 2.8 \\
\hline & Present, Left and Right TMJs & 7 & 2.0 \\
\hline
\end{tabular}

Assessment of salivary cortisol levels between the TMD and control groups, as seen in Table 3, showed a statistically significant $(\mathrm{p}<0.001)$ difference. Evaluation of the stress levels also revealed a statistically significant $(\mathrm{p}<0.001)$ difference between the control and TMD groups, as seen in Table 4.

Table 3. Salivary Cortisol Levels between TMD patients and control.

\begin{tabular}{cccc}
\hline Salivary Cortisol Levels & N & Mean (SD) & p-value \\
\hline TMD Patients & 20 & $1.107 \pm 0.17$ & $<0.001^{*}$ \\
Control & 20 & $0.696 \pm 0.16$ & \\
*Unpaired t-test. & & &
\end{tabular}

Table 4. Stress levels between TMD patients and controls.

\begin{tabular}{|c|c|c|c|c|}
\hline Stress Scores & $\mathbf{N}$ & Mean Rank & Sum of Ranks & p-value \\
\hline TMD Patients & 20 & 60.5 & 2420 & $<0.001^{*}$ \\
\hline Control & 20 & 20.5 & 820 & \\
\hline Total & 40 & & & \\
\hline
\end{tabular}

The salivary cortisol levels were compared between the sexes in the control and TMD groups. A statistically significant difference $(\mathrm{p}<0.05)$ was seen in the cortisol levels between the male and female students in both the control and TMD groups (Table 5).

Table 5. Salivary cortisol levels between control group and TMD group according to gender.

\begin{tabular}{lccc}
\multicolumn{1}{c}{ Group } & Mean & SD & p-value \\
\hline Control - Females & 0.786 & 0.117 & $0.0129^{*}$ \\
Control - Males & 0.607 & 0.157 & \\
TMD - Females & 1.227 & 0.158 & $0.0015^{*}$ \\
TMD - Males & 0.987 & 0.0632 & \\
\hline
\end{tabular}

*Paired t-test. 
Similarly, the stress levels were compared between the sexes in the control and TMD groups in Table 6, and a statistically significant difference $(\mathrm{p}<0.05)$ was seen in the stress levels between males and female students in both the control and TMD groups.

Table 6. Stress levels between control group and TMD group according to gender.

\begin{tabular}{lccc}
\hline \multicolumn{1}{c}{ Group } & Mean & SD & p-value \\
\hline Control - Females & 9.1 & 2.024 & $0.0338^{*}$ \\
Control - Males & 6.8 & 2.043 & \\
TMD - Females & 29.1 & 3.928 & $0.00186^{*}$ \\
TMD - Males & 24.8 & 4.779 & \\
*Paired t-test. & & &
\end{tabular}

The students diagnosed with stress were categorized as low, moderate, or high. In our study, all the students in the control group showed low stress (100\%) levels, while the students in the TMD group showed moderate $(45 \%)$ and high-stress levels $(55 \%)$, as seen in Table 7 . A moderate positive correlation was observed $(\mathrm{r}=0.561)$ between the salivary cortisol and stress levels in TMD group.

Table 7. Categorization of control group and TMD group based on PSS scores.

\begin{tabular}{lcc}
\hline \multicolumn{1}{c}{ Stress Levels } & Control Group $(\mathbf{n = 2 0})$ & TMD Group $(\mathbf{n = 2 0})$ \\
\hline Low Stress & 20 & - \\
Moderate Stress & - & 9 \\
High Stress & - & 11 \\
\hline
\end{tabular}

\section{Discussion}

The TMJ disorder is a commonly occurring jaw-associated problem. Patients with TMD generally experience joint and muscle pain during mandibular movements, joint sounds, and a limited range of mandibular motion. TMD can present with varying degrees of signs and symptoms affecting individuals of any age group and sex [Q-4]. The validity and reliability of the techniques in the RDC/TMD questionnaire for diagnosis of TMD have been critically assessed for their use in clinical settings and research purposes $[2,10,19]$. Symptoms of stress usually present as clinical manifestations of TMDs, resulting in changes in the levels of cortisol secretion. Hence, salivary cortisol and stress levels were assessed in patients with TMD. The students with TMD presented with higher stress levels and higher cortisol levels. The hypothesis that students with TMD did not present with any changes in stress and salivary cortisol levels could not be accepted.

The prevalence of TMD in this study was $30.7 \%$. The sample consisted of $53.7 \%$ female and $46.2 \%$ male students. Concerning sex, female students (57\%) presented a higher prevalence of TMD than male students (43\%). Ahmed et al. [23] reported similar results in a study on 500 Sudanese students in which females presented a higher prevalence of TMD than males. Similar results were reported by Oliveira et al. [25] in a study on 2396 Brazilian students in which 73.03\% of females exhibited TMD compared to 56.26\% of males. Karthik et al. [26] reported that the prevalence of TMD in females was almost twice that in males. This higher prevalence in females can be explained by physiological factors, such as variations in hormonal levels, connective tissue characteristics, and muscle structure [25,26].

In our study, the RDC/TMD Axis I showed that the participants presented with a higher prevalence of muscle disorders (group I), seen in $14.2 \%$ of the participants. The prevalence of disk displacement (group II) was seen in $5.6 \%$ of the participants with a reduction on the right/left/both TMJs. Arthralgia, osteoarthritis, 
or osteoarthrosis (group III) was seen in $4.8 \%$ of the participants. Salameh et al. [27] reported that group I muscle disorders were the most common and were detected in $56.7 \%$ of patients, whereas disk displacement in 26.7\%, and arthralgia, osteoarthritis, or osteoarthrosis were detected in $16.7 \%$. Furthermore, a higher prevalence of TMD was observed in females than in males (females 70\% and males 30\%) [27]. A study by Progiante et al. [28] assessed the prevalence of TMDs in the Brazilian population using the RDC/TMD Axis I questionnaire and reported that the prevalence of muscle disorders (group I) was 29.5\%, disk displacement (group II) was 7.9\%, and arthralgia, osteoarthritis, or osteoarthrosis (group III) was 39.1\%. A meta-analysis for $\mathrm{RDC} / \mathrm{TMD}$ Axis I prevalence in a systematic review reported the prevalence of muscle disorders (group I) was $45.3 \%$, disk displacement (group II) was $41.1 \%$, and arthralgia, osteoarthritis, or osteoarthrosis (group III) was $30.1 \%$ [2]. The results of this study are consistent with those of a study conducted in the Brazilian population that used the RDC/TMD questionnaire in patients with TMD and reported the prevalence of muscle disorders in $77.1 \%$ of the population, disk displacements in $75.7 \%$, and other joint disorders in $61.4 \%$ of the population [29]. However, the prevalence rate of TMD was higher in the two previous studies than in our study, owing to the larger sample size.

An association between stress and presence of painful TMD has been established. Generally, stress can trigger hyperactivity of the muscle and pain, leading to joint inflammation [30]. After evaluating the PSS, stress levels were found to be increased in the TMD group than in the control group. In this study, a statistically significant difference $(\mathrm{p}<0.05)$ was observed between the male and female students' stress levels in the control and TMD groups. All the students in the control group exhibited low-stress levels; whereas, students in the TMD group exhibited moderate and high-stress levels. In our study, $45 \%$ of the students in the TMD group had moderate stress and 55\% had high stress levels. Salameh et al. [27] also reported higher stress levels in TMD cases than in the controls with the PSS, similar to this study.

Salivary cortisol estimation was considered over plasma cortisol, as it is a non-invasive procedure and results are similar to those of plasma cortisol. Following the circadian rhythm of cortisol secretion, saliva was collected in the morning, as levels of the hormone are higher [23]. In this study, cortisol levels were higher in the TMD group. The cortisol levels in saliva varied from 0.8 to $1.0 \mu \mathrm{g} / \mathrm{dL}$ for the TMD group and from 0.4 to $0.9 \mu \mathrm{g} / \mathrm{dL}$ for the control group. The mean and standard deviation of salivary cortisol levels for TMD and control groups were $1.107 \pm 0.17$ and $0.696 \pm 0.16$, respectively. The cortisol levels in the TMD group were higher than the normal levels $(0.3-1 \mu \mathrm{g} / \mathrm{dL})$. The difference in the cortisol levels between the TMD and control groups was statistically significant $(\mathrm{p}<0.001)$. The results obtained were similar to those of Salameh et al. [27]. Significant differences in the salivary cortisol concentrations between the TMD group and controls were observed [26]. Similar results were observed in a study by Ali et al. [31], where the TMD group with stress showed significantly higher levels of saliva cortisol $(177.8 \pm 12.24 \mathrm{ng} / \mathrm{mL})$ than the control $(22.9 \pm 2.28$ $\mathrm{ng} / \mathrm{mL}$ ). A statistically significant difference was seen between the cortisol values among the male and female students in the control and TMD groups. A positive correlation was observed between stress and salivary cortisol levels among students with TMD

The limitation of this study was the consideration of a small sample size for the estimation of cortisol and stress levels. It impaired the comparison of cortisol levels in low, moderate, and high stress levels. Future studies with a larger sample size are recommended, which may help to strengthen the concept of using salivary cortisol levels as a biomarker for the diagnosis of stress in individuals with TMD. 


\section{Conclusion}

This study showed that psychosocial stress played a vital role in the etiopathogenesis of temporomandibular disorders. Female students were at a higher risk of TMD than male students, and muscle disorders were the most common. The significantly higher levels of salivary cortisol and stress levels in individuals with TMD indicated the significance of salivary cortisol levels as a biological marker of stress in patients with temporomandibular disorders.

\section{Authors' Contributions}

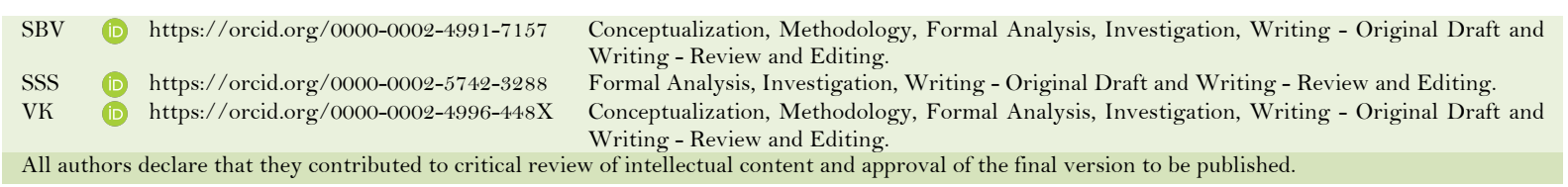

\section{Financial Support}

None.

\section{Conflict of Interest}

The authors declare no conflicts of interest.

\section{Data Availability}

The data used to support the findings of this study can be made available upon request to the corresponding author.

\section{Acknowledgments}

The authors thank all the students who volunteered to participate in the study. The authors sincerely thank Mr. Chung Luo Wei, Mr. Kishen a/1 Balakrishnan and Ms. Rangiita A/P Govindarajoo for their valuable contribution in data collection.

\section{References}

[1] McNeill C. Management of temporomandibular disorders: concepts and controversies. J Prosthet Dent 1997; 77(5):510-22. https://doi.org/10.1016/So022-3913 (97)70145-8

[2] Manfredini D, Guarda-Nardini L, Winocur E, Piccotti F, Ahlberg J, Lobbezoo F. Research diagnostic criteria for temporomandibular disorders: a systematic review of axis I epidemiologic findings. Oral Surg Oral Med Oral Pathol Oral Radiol Endod 2011; 112(4):453-62. https://doi.org/10.1016/j.tripleo.2011.04.021

[3] Hongxing L, Astrom AN, List T, Nilsson IM, Johansson A. Prevalence of temporomandibular disorder pain in Chinese adolescents compared to an age-matched Swedish population. J Oral Rehabil 2016; 43(4):241-8. https://doi.org/10.1111/joor.12366

[4] Kobayashi FY, Gavião MBD, Marquezin MCS, Fonseca FLA, Montes ABM, Barbosa TS, et al. Salivary stress biomarkers and anxiety symptoms in children with and without temporomandibular disorders. Braz Oral Res 2017; 31:e78. https://doi.org/10.1590/1807-3107BOR-2017.vol31.0078

[5] Schiffman EL, Fricton JR, Haley DP, Shapiro BL. The prevalence and treatment needs of subjects with temporomandibular disorders. J Am Dent Assoc 1990; 120(3):295-303. https://doi.org/10.14219/jada.archive.1990.0059

[6] Carlson GE, Magnusson T. Management of Temporomandibular Disorders in the General Dental Practice. London: Quintessence Publishing Co. Inc.; 1990.

[7] Carlsson GE. Epidemiology and treatment need for temporomandibular disorders. J Orofac Pain 1999;13(4):232-7.

[8] Solberg WK, Woo MW, Houston JB. Prevalence of mandibular dysfunction in young adults. J Am Dent Assoc 1979; 98(1):25-34. https://doi.org/10.14219/jada.archive.1979.0008

[9] Pedroni CR, De Oliveira AS, Guaratini MI. Prevalence study of signs and symptoms of temporomandibular disorders in university students. J Oral Rehabil 2003; 30(3):283-9. https://doi.org/10.1046/j.1365-2842.2003.01010.x

[10] Zulqarnain BJ, Khan N, Khattab S. Self-reported symptoms of temporomandibular dysfunction in a female university student population in Saudi Arabia. J Oral Rehabil 1998; 25(12):946-53.

https://doi.org/10.1046/j.1365-2842.1998.00334.x 
[11] Dworkin SF, LeResche L. Research diagnostic criteria for temporomandibular disorders: review, criteria, examinations and specifications, critique. J Craniomandib Disord 1992; 6(4):301-55.

[12] Campos JADB, Goncalves DAG, Camparis CM, Speciali JG. Reliability of a form for diagnosing the severity of temporomandibular disorder. Rev Bras Fisioter 2009; 13(1):38-43.

https://doi.org/10.1590/S1413-35552009005000007

[13] Schmitter M, Ohlmann B, John MT, Hirsch C, Rammelsberg P. Research diagnostic criteria for temporomandibular disorders: a calibration and reliability study. Cranio 2005; 23(3):212-8. https://doi.org/10.1179/crn.2005.030

[14] John MT, Dworkin SF, Mancl LA. Reliability of clinical temporomandibular disorder diagnoses. Pain 2005; $118(1-$ 2):61-9. https://doi.org/10.1016/j.pain.2005.07.018

[15] List T, John MT, Dworkin SF, Svensson P. Recalibration improves inter-examiner reliability of TMD examination. Acta Odontol Scand 2006; 64(3):146-52. https://doi.org/10.1080/00016350500483137

[16] Greenberg MS, Glick M. Burket's Oral Medicine Diagnosis \& Treatment. 10th ed. Hamilton: BC Decker; 2003.

[17] Wu G, Chen L, Zhu G, Su, Y, Chen Y, Sun J, et al. Psychological stress induces alterations in temporomandibular joint ultrastructure in a rat model of temporomandibular disorder. Oral Surg Oral Med Oral Pathol Oral Radiol Endod 2011; 112(6): e106-e112. https://doi.org/10.1016/j.tripleo.2011.06.005

[18] Miller GE, Chen E, Zhou ES. If it goes up, must it come down? Chronic stress and the hypothalamic-pituitaryadrenocortical axis in humans. Psychol Bull 2007; 133(1):25-45. https://doi.org/10.1037/0033-2909.133.1.25

[19] Laccio KJD, Colato AS, Dorneles G, Peres A. Assessment of levels of salivary cortisol and stress in patients with signs and symptoms of temporomandibular joint disorders. Int $\mathrm{J}$ Health Sci 2014; 2(4):59-72. https://doi.org/10.15640/ijhs.v2n4a5

[20] Luther F, Layton S, McDonald F. Orthodontics for treating temporomandibular joint (TMJ) disorders. Cochrane Database Syst Rev 2010; (7):CD006541. https://doi.org/10.1002/14651858.CD006541.pub2

[21] Vining RF, McGinley RA. The measurement of hormones in saliva: possibilities and pitfalls. J Steroid Biochem 1987; 27(1-3):81-94. https://doi.org/10.1016/0022-4731(87)90297-4

[22] Weibel L. Methodological guidelines for the use of salivary cortisol as biological marker of stress. Presse Med 2003; 32(18):845-51.

[23] Ahmed LI, Abuaffan AH. Prevalence of temporomandibular joint disorders among Sudanese university students. J Oral Hyg Health 2016; 4(2):200. https://doi.org/10.4172/2330702.1000202

[24] Okano K, Kaczmarzyk JR, Dave N, Gabrieli JDE, Grossman JC. Sleep quality, duration, and consistency are associated with better academic performance in college students. NPJ Sci Learn 2019; 4:16. https://doi.org/10.1038/s41539-019-0055-Z

[25] Oliveira AS, Dias EM, Contato RG, Berzin F. Prevalence study of signs and symptoms of temporomandibular disorder in Brazilian college students. Braz Oral Res 2006; 20(1):3-7.

https://doi.org/10.1590/S1806-83242006000100002

[26] Karthik R, Hafila MIF, Saravanan C, Vivek N, Priyadarsini P, Ashwath B. Assessing prevalence of temporomandibular disorders among university students: a questionnaire study. J Int Soc Prev Community Dent 2017; 7(Suppl 1):S24-S29. https://doi.org/10.4103/jispcd. JISPCD_146_17

[27] Salameh E, Alshaarani F, Hamed HA, Nassar JA. Investigation of the relationship between psychosocial stress and temporomandibular disorder in adults by measuring salivary cortisol concentration: a case-control study. J Indian Prosthodont Soc 2015; 15(2):148-52. https://doi.org/10.4103/0972-4052.158075

[28] Progiante PS, Pattussi MP, Lawrence HP, Goya S, Grossi PK, Grossi ML. Prevalence of temporomandibular disorders in an adult brazilian community population using the Research Diagnostic Criteria (Axes I and II) for Temporomandibular Disorders (The Maringá Study). Int J Prosthodont 2015; 28(6):600-9. https://doi.org/10.11607/ijp.4026

[29] Barros VM, Seraidarian PI, Côrtes MI, de Paula LV. The impact of orofacial pain on the quality of life of patients with temporomandibular disorder. J Orofac Pain 2009; 23(1):28-37.

[30] Nilsson AM, Dahlström L. Perceived symptoms of psychological distress and salivary cortisol levels in young women with muscular or disk-related temporomandibular disorders. Acta Odontol Scand 2010; 68(5):284-8. https://doi.org/10.3109/00016357.2010.494620

[31] Ali QS, Hadi R. Assessment of cortisol as salivary psychological stress marker in relation to temporomandibular disorders among a sample of dental students. JBCD 2015; 27(2):86-92. 